\title{
Stakeholders' Recommendations to Improve Patient-centered "LGBTQ" Primary Care in Rural and Multicultural Practices
}

\author{
Miria Kano, PhD, Alma Rosa Silva-Bañuelos, BA, Robert Sturm, $M A$, and \\ Catbleen E. Willging, PhD
}

Purpose: Individuals among gender/sexual minorities share experiences of stigma and discrimination, yet have distinctive health care needs influenced by ethnic/racial minority and rural realities.

Methods: We collected qualitative data from lesbian/gay/bisexual/transgender (LGBT) and queer persons across the largely rural, multicultural state of New Mexico, particularly those from understudied ethnic groups, regarding factors facilitating or impeding patient-centered primary care. The themes identified formed the basis for a statewide summit on LGBT health care guidelines and strategies for decreasing treatment gaps.

Results: Three to 15 individuals, ages 18 to 75 years, volunteered for 1 of 4 town hall dialogues $(\mathrm{n}=32)$, and 175 people took part in the summit. Participants acknowledged health care gaps pertinent to LGBT youth, elders, American Indians, and Latinos/Latinas, expressing specific concern for rural residents.

Conclusions: This preliminary research emphasizes the need to improve primary care practices that treat rural and ethnic-minority LGBT people and offers patient-driven recommendations to enhance care delivery while clinic-level transformations are implemented. (J Am Board Fam Med 2016;29: 156-160.)

Keywords: Bisexuals, Gays, Health Planning Guidelines, Lesbians, Minority Health, Primary Health Care, Rural Health, Transgenders

Reducing health care disparities for lesbian, gay, bisexual, transgender, and queer/questioning (LGBTQ) people is a major public health priority. ${ }^{1-4}$ Compared with heterosexual, cisgender

This article was externally peer reviewed.

Submitted 30 June 2015; revised 2 October 2015; accepted 7 October 2015.

From the Department of Family and Community Medicine, University of New Mexico, Albuquerque (MK); the University of New Mexico LGBTQ Resource Center, Albuquerque (ARS-B); the New Mexico Community AIDS Partnership, Santa Fe (RS); and the Pacific Institute for Research and Evaluation, Behavioral Health Research Center of the Southwest, Albuquerque, NM (CEW).

Funding: This project was funded through a Patientcentered Outcomes Research Institute (PCORI) Pipelineto-Proposal Award (MK), administered on behalf of PCORI by the Colorado Foundation for Public Health and the Environment.

Conflict of interest: none declared.

Corresponding author: Miria Kano, PhD, Department of Family and Community Medicine, University of New Mexico, MSC09 5040, 2400 Tucker St NE, Albuquerque, NM 87131-0001 (E-mail: mkano@salud.unm.edu). populations, LGBTQ people suffer from higher rates of mental health and substance use problems, eating and body-related disorders, and sexually transmitted diseases ${ }^{5}$; are at greater risk for poor diet and insufficient exercise ${ }^{6,7}$; and are less likely to benefit from preventive services (eg, cancer screens) and treatment for comorbid conditions (eg, cardiovascular disease and diabetes). ${ }^{7}$ Furthermore, isolated LGBTQ persons in rural and/or ethnic minority communities may face stigma and unique challenges in accessing care that is responsive to their treatment needs. ${ }^{8}$

Primary care providers, particularly those in rural and underserved areas, are the front line of vital prevention, screening, and treatment services for patients throughout the life course. Yet few primary care clinics have established systems in place to create practice environments that respect LGBTQ patients as a group. ${ }^{9}$ While recent efforts to increase equity for LGBTQ individuals include 
supportive national health policies, recommendations for culturally appropriate care,${ }^{10}$ and specialized medical education curricula, ${ }^{11}$ such policies/ curricula are not widely integrated within clinics, and targeted interventions to enhance LGBTQ health are in the nascent stages of development. ${ }^{2} \mathrm{~A}$ 2010 systematic review of treatment guidelines for LGBTQ patients called for greater primary care research and interventions. ${ }^{12}$ The few guidelines available ignored population-based attributes of LGBTQ people (eg, demographic and cultural), failed to acknowledge rural environs, and were considered most effective when accompanied by "audits, training and feedback" mechanisms that are difficult to implement in understaffed and underresourced primary care practices. ${ }^{13} \mathrm{We}$ conducted a pilot study to bring attention to the experiences of LGBTQ persons in rural, understudied, minority communities in receiving primary care and the degree to which such guidelines could possibly improve services in these clinical settings.

\section{Methods}

Between March and August 2014, we used semistructured interview guides to engage LGBTQ persons across New Mexico in town hall dialogues to explore factors affecting optimal patient-centered health care. Community advocate partners reviewed recruitment materials and helped to recruit a range of LGBTQ individuals from 4 small urban centers with access to neighboring rural locales for participation in town hall dialogues. The dialog format allowed us to recruit broadly, publicizing the meeting/venue to sizeable numbers of LGBTQ persons without requiring a reply or signup-actions with the potential to compromise the confidentiality of those interested in attending. Community partners reviewed discussion questions, ensuring that the language used was culturally appropriate and accessible for people of varying literacy levels.

We explained informed consent using a script approved by the University of New Mexico Institutional Review Board; all participants gave verbal consent for participation. Two researchers (MK and CEW) moderated each town hall, asking open-ended questions focused on perceptions of LGBTQ primary care within communities, patient comfortseeking services, cultural appropriateness of care, providers and/or services that encourage LGBTQ health, service gaps for LGBTQ patients, and suggestions to improve LGBTQ health locally. We digitally recorded and transcribed these dialogues, importing them into the NVivo 10 qualitative data analysis software (QSR International, Burlington, MA), for iterative coding and analysis and efficient text retrieval. Members of the research team independently reviewed transcripts, identified issues that cross-cut the town hall dialogues, and agreed on interpretations.

The research team presented the issues at a subsequent statewide event entitled, "New Mexico's Sexual and Gender Diversity Summit: New Directions for LGBTQ Health and Wellbeing." By sharing the findings from the dialogues, the team intended to establish a context for discussions of LGBTQ health disparities during the summit. Summit attendees also identified key populations affected by health disparities that could serve as a focal point for critical reflection during facilitated sessions based on open space technology. ${ }^{14}$ During these sessions, attendees were encouraged to suggest strategies that could be applied to ameliorate these particular disparities. Participant discussions were guided by the following questions:

1. What barriers prevent health equity for this population and/or what issues need to be addressed?

2. What are the desired health outcomes for the identified population?

3. What factors are in place to facilitate these outcomes?

4. What actions steps can be taken to improve LGBTQ primary care for this population?

Attendees were informed that participation in summit activities was voluntary, and that information arising from the process would be used for research development, publication, and to promote equitable health policies for LGBTQ New Mexicans. Session responses were hand-recorded and compiled into a report that was distributed to summit participants.

\section{Results}

Three to 15 individuals, ages 18 to 75 years, volunteered for each local/rural dialog $(\mathrm{n}=32)$. Participants reported anti-LGBTQ sentiment that led some LGBTQ people to "pocket" or closet them- 
Table 1. Characteristics of Summit Participants by Organizational Affiliation and Geographic Location

\begin{tabular}{lcc}
\hline & \multicolumn{2}{c}{ Participants (n) } \\
\cline { 2 - 3 } Organizational Affiliation & Urban & Rural \\
\hline $\begin{array}{l}\text { Community member (no reported } \\
\quad \text { organizational affiliation) }\end{array}$ & 29 & 14 \\
Health care provider & 31 & 13 \\
LGBTQ health advocate/educator & 24 & 4 \\
High school/university educator & 5 & 2 \\
Student & 15 & 11 \\
Researcher & 13 & 4 \\
State department staff & 7 & 0 \\
Policymaker & 3 & 0 \\
\hline
\end{tabular}

LGBTQ, lesbian/gay/bisexual/transgender/queer.

selves, a persistent lack of culturally sensitive health care, deficits in provider knowledge and/or lack of provider inquiry into LGBTQ-specific health issues, fragmentation of health care services (particularly for persons with HIV), insufficient health care options for rural LGBTQ patients, and isolation of youth, elders, and transgender persons. A comprehensive description of town hall findings was presented in the opening address for the New Mexico Sexual and Gender Diversity Summit. The 175 summit attendees (see Table 1 for demographic information) identified additional health care gaps for 7 populations: LGBTQ youth, elders, American Indians, Latinos/Latinas (native and immigrant), transgender persons, bisexual people, and all LGBTQ rural residents. Quotations derived from the town hall dialogues reflecting dominant perceptions/experiences, as well as a summary of summit participant suggestions to address gaps in the health care system, are presented here.

One urban dialogue participant's comment captured the tone of these conversations: "It is not good here. But I have access to care if I need it. Imagine being a trans woman of color in Portales!" For this participant, Portales, a rural community of 12,233 people, $43 \%$ of whom are Hispanic, exemplified New Mexico communities shaped by specific cultural contexts and the lack of resources for LGBTQ-centered health care. Many rural participants reported struggles with "fear" and "anxiety" due to perceived discrimination and bias during health care encounters, and claimed to omit relevant information about themselves as a strategy to ensure access to care and avoid staff/provider discomfort. One gay participant told us, "Here, it is better not to disclose your sexuality. My partner went to the emergency department for gastrointestinal problems. He could not get a hold of me and was totally on his own. When the nurses found out he was gay, they left him for hours. . . Homophobia is real here. How do you think they reacted when they found out he was HIV positive?"

To meet such needs, summit attendees identified primary care as a key setting for first-level interventions because of its person-centeredness and accessibility for rural and economically challenged New Mexicans. When asked whether extant primary care treatment guidelines would be helpful to share with clinicians statewide, the majority were unaware that such guidelines existed, whereas those in the know indicated that the guidelines did not focus sufficiently on the needs of rural gender/ sexual minorities.

Summit attendees suggested that clinics may be unaware of and/or lack sufficient resources to integrate LGBTQ-specific care recommendations into practice. A lesbian who worked for a managed care organization explained, "It is not that providers are bad people, or want to be insensitive. There are always going to be those whose views are homophobic, or overtly religious and conservative. But others just haven't had training, and do not know what to say to a male-to-female transgender patient who was just beaten for walking down the street or having a drink in a local bar."

Summit participants believed strongly that staff and clinicians would benefit from LGBTQ health care training and evaluation, indicating that guidelines calling for full cultural competency coaching for all staff/providers would increase sensitivity, confidentiality, and positive interactions for LGBTQ patients. A gay man who participated in a dialogue explained, "I want them to ask me. I want them to offer anal paps. But, the docs are uncomfortable talking about sex, much less talking about gay sex."

Summit participants pointedly appealed for "realistic action steps" that could lead to "immediate improvements" for LGBTQ patient-centered care:

1. Create a safe, welcoming environment

- Post LGBTQ-positive visual representations, for example, images of people/families of differing gender/sexual, cultural, ethnic/racial backgrounds, in lobbies/examination rooms 
- Use culturally appropriate, culturally specific terms on intake forms; that is, recognize that some patients may prefer local terminologies such as Native American use of "Two-Spirit," patient preference for "queer" over "lesbian," "gay" or "bisexual," and/or use of "they" as a preferred gender pronoun by people across the sexual/gender minority spectrum

- Include gender/sexual difference in displayed antidiscrimination policies

\section{Develop culturally competent medical staff}

- Invite local LGBTQ community members/leaders to clinic meetings to discuss the experiences/ treatment needs of LGBTQ patients

- Encourage respectful, direct, open-ended questions using culturally relevant terminology in provider-patient interactions regarding identity, sexual history, and risk factors

- Use local university/community-based safe zone training ${ }^{15}$ centered on how to be an LGBTQ ally while enhancing understanding of anti-LGBTQ stigma/phobias and microaggressions for all providers and staff to encourage patient feelings of security

\section{Update medical training}

- Deploy technologies in rural settings, for example, video/Web conferencing for consulting LGBTQ specialists

- Acquire information on LGBTQ-specific health, for example, hormone therapies and associated risks for transgender patients, gender-sensitive cancer screenings

- Participate in research to improve LGBTQ health care

\section{Discussion}

The concerns of gender/sexual minorities who participated in this brief pilot study call attention to the reality that LGBTQ-supportive primary care is uneven and driven by locale and resources. While LGBTQ populations in many areas benefit from the practices of highly skilled clinicians and their primary care staff-like the care provided through the Fenway Institute, a prominent center for LGBTQ health research, training, and policy in Boston, MA—such expertise and resources do not necessarily influence practice culture in primary care sites in rural and underserved areas in New Mexico. Providers in these remote regions have smaller, yet no less significant, LGBTQ patient bases, and fewer opportunities for training and engagement designed specifically for single-provider and/or small practices that might lead to enhanced service delivery for this vulnerable population.

The providers, community members, and health advocates who participated in this summit felt that a list of relatively inexpensive yet meaningful interventions could have a profound influence on rural primary care practices that lack resources and knowledge about providing specific treatment for LGBTQ patients, eliminating concerns regarding the propensity of many gender/sexuality minorities to not disclose information about their sexuality to providers. This is a significant point: previous research indicates that some providers believe that nondisclosure indicates that gender/sexuality is "unimportant" to patients ${ }^{16}$ or, conversely, that their lack of inquiry reflects a "neutral" attitude toward LGBTQ patients, a treatment strategy that some feel masks provider discomfort or negative beliefs about gender/sexual minorities. ${ }^{17}$ Studies find that providers' inability to communicate effectively with patients results in poorer health care. ${ }^{18}$ While existing guidelines encourage affirmative care for LGBTQ patients, ${ }^{12}$ they generally fall short in describing the pragmatics of facilitating patient disclosure, a measure likely to reduce health care disparities for gender/sexual minorities, ${ }^{12}$ and are perceived by the participants of this study to be inadequate for addressing the unique circumstances of rural ethnic minority patients and primary care practices burdened by resource and time constraints.

Limitations of this work include its geographic specificity and patient-level focus. However, this work recognizes gaps in both LGBTQ health care guidelines and rural primary care requiring further investigation. It also highlights the need to integrate perspectives of both patients and primary care providers in all aspects of study design, implementation, and evaluation to ensure interest in, relevance of, and sustainment of LGBTQ-related health care interventions in underserved areas.

The authors thank Patricia Hokanson for her support of this manuscript, and members of the New Mexico LGBTQ Health Collaborative, namely Edward Fancovic, Rebecca Dakota, Am- 
ber Royster, Jonathan Flores, Paige Duhamel, Cameron Crandall, Nathaniel Sharon, La Tisha Rico, Molly Adler, Beverly Gorman, Benjamin Moser, Adrien Lawyer, Jamie Finkelstein, Crystal Romney, and Denise Ruybal, for their work to improve the health of LGBTQ New Mexicans, and their assistance with participant recruitment, summit sponsorship, and meeting facilitation that made these recommendations possible.

\section{References}

1. Office of Disease Prevention and Health Promotion. Healthy People 2020 topics \& objectives. Washington, DC: U.S. Department of Health and Human Services; 2015. Available from: http://www.healthypeople.gov/2020/topicsobjectives2020/default. Accessed March 12, 2015.

2. Institute of Medicine. The health of lesbian, gay, bisexual, and transgender people: building a foundation for better understanding. Washington, DC: $\mathrm{Na}-$ tional Academies Press; 2011.

3. Ward BW, Dahlhamer JM, Galinsky AM, Joestl SS. Sexual orientation and health among U.S. adults: National Health Interview Survey, 2013 (no. 77). Hyattsville, MD: National Center for Health Statistics; 2014.

4. VanKim NA, Padilla JL. New Mexico's progress in collecting lesbian, gay, bisexual, and transgender health data and its implications for addressing health disparities. Albuquerque: New Mexico Department of Health Chronic Disease Prevention and Control Bureau; 2010.

5. Meyer IH. Identity, stress, and resilience in lesbians, gay men, and bisexuals of color. Counsel Psychol 2010;38:442-54.

6. Fredriksen-Goldsen KI, Cook-Daniels L, Kim H, et al. Physical and mental health of transgender older adults: an at-risk and underserved populations. Gerontologist 2013;54:488-500.

7. Molina Y, Lehavot K, Beadnell B, Simoni J. Racial disparities in health behaviors and conditions among lesbian and bisexual women: the role of internalized stigma. LGBTQ Health 2014;1:131-9.

8. Willging CE, Salvador M, Kano M. Pragmatic help seeking: how sexual and gender minority groups access mental health care in a rural state. Psychiatr Serv 2006;57:871-4.
9. Eliason MJ, Schope R. Does "don't ask don't tell" apply to health care? Lesbian, gay, and bisexual people's disclosure to health providers. J Gay Lesbian Med Assoc 2010;5:125-34.

10. U.S. Department of Health and Human Services. Better health and well-being: making improvements for lesbian, gay, bisexual, transgender (LGBTQ) Americans. Washington, DC: U.S. Department of Health and Human Services; 2012.

11. American Academy of Family Physicians, Society of Teachers of Family Medicine Group on LGBTQ Health. Recommended curriculum guidelines for family medicine residents: lesbian, gay, bisexual, transgender health. AAFP reprint no. 289D. Leawood, KS: American Academy of Family Physicians. Available from: http://www.aafp.org/ dam/AAFP/documents/medical_education_residency/program_directors/Reprint289D_LGBT.pdf. Acessed June 22, 2015.

12. McNair RP, Hegarty K. Guidelines for the primary care of lesbian, gay, and bisexual people: a systematic review. Ann Fam Med 2010;8:533-41.

13. Davy Z, Siriwardena AN. To be or not to be LGBTQ in primary health care: health care for lesbian, gay, bisexual, and transgender people. Br J Gen Pract 2012;62:491-2.

14. Herman, Michael. What is open space technology? Available from: http://openspaceworld.org/wp2/ what-is/. Accessed June 22, 2015.

15. University of New Mexico LGBTQ Resource Center. Safe zone training. Available from: http:// lgbtqrc.unm.edu/services/safe-zone-trainings.html. Accessed June 22, 2015.

16. Sherman MD, Kauth MR, Shipherd JC, Street RL. Provider beliefs and practices about assessing sexual orientation in two Veterans Health Affairs hospitals. LGBTQ Health 2014;1:185-91.

17. Willging CE, Salvador M, Kano M. Unequal treatment: mental health care for sexual and gender minority groups in a rural state. Psychiatr Serv 2006; 57:867-70.

18. Petroll AE, Mosack KE. Physician awareness of sexual orientation and preventive health recommendations to men who have sex with men. Sex Transm Dis 2011;38:63-7. 